
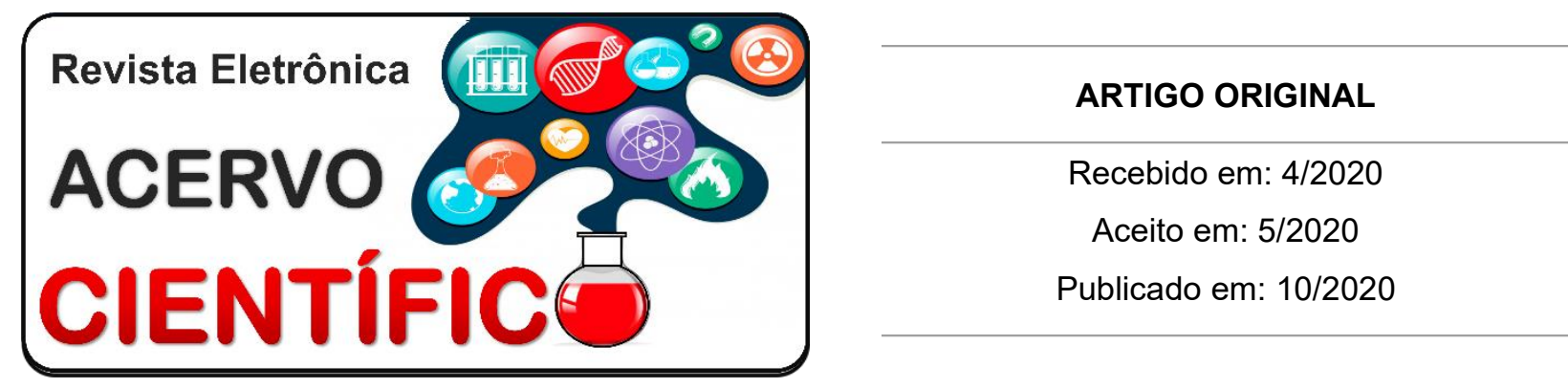

\title{
Uso de psicoestimulantes por estudantes de medicina em uma faculdade particular de Juiz de Fora - MG
}

\author{
Use of psychostimulant among medical students from a private college of Juiz de Fora - \\ MG
}

El uso de psicostimulantes entre los estudiantes de medicina de un faculdad privado de Juiz de Fora - MG

Amanda Borges Teixeira ${ }^{1 *}$, Bruna Reboredo Lôbo ${ }^{1}$, Gustavo de Paula Paiva ${ }^{1}$, João Otávio de Souza Carvalho', Laura Brandão Lemos ${ }^{1}$, Leticia Nunes Arantes Fuhr ${ }^{1}$, Lília Alves dos Santos ${ }^{1}$, Guilherme Henrique Faria do Amaral ${ }^{1}$, Nathália Barbosa do Espírito Santo Mendes ${ }^{1}$, Guillermo Patricio Ortega Jácome'.

Resumo: O presente trabalho almejou verificar o uso de psicoestimulantes por parte dos acadêmicos de medicina de uma faculdade particular de Juiz de Fora. Foi realizado um estudo observacional do tipo transversal com 213 alunos acadêmicos de medicina do $1^{\circ}$ ao $12^{\circ}$ períodos, com exclusão dos menores de 18 anos. Os dados foram coletados por meio do questionário: "Adult Self-Report Scale", onde o uso de psicoestimulantes foi constatado em $31,5 \%$ dos entrevistados. Ainda, dentre os que não utilizaram, $31,5 \%$ reportaram interesse prévio no uso. A maioria dos usuários era do sexo feminino $(52,2 \%)$, sendo que $62,7 \%$ dos acadêmicos que alegaram uso de psicoestimulantes não possuíam diagnóstico prévio de Transtorno de Déficit de Atenção e Hiperatividade (TDAH). Portanto, observa-se a relevância da compreensão do padrão de uso atual de psicoestimulantes por universitários, corroborando para possíveis estratégias de melhora da saúde mental relacionadas ao tema.

Palavras-chave: Transtorno do Deficit de Atenção com Hiperatividade, Estudantes, Epidemiologia.

\begin{abstract}
Abstrac: The present study aimed to verify the use of psychostimulants among medical students from a private college of Juiz de Fora. A cross-sectional observational study was carried out with 213 medical students from 1st to 12 th $^{\circ}$ periods, excluding minors under 18 years old. Data were collected through the questionnaire: "Adult Self-Report Scale", where the use of psychostimulants was found in $31.5 \%$ of the interviewees. Still, among those who did not use it, $31.5 \%$ reported previous interest in the use. The majority of users were female $(52.2 \%)$, and $62.7 \%$ of the students who claimed to use psychostimulants did not have a previous diagnosis of Attention Deficit Hyperactivity Disorder (ADHD). Therefore, the relevance of understanding the pattern of current use of psychostimulants by university students is observed, corroborating possible strategies for improving mental health related to the theme.
\end{abstract}

Keywords: Attention Deficit Disorder with Hyperactivity, Students, Epidemiology.

1 Universidade Presidente Antônio Carlos (UNIPAC), Juiz de Fora - MG. *E-mail: amandabt@live.com 
Resumen: El presente estudio apuntó a verificar el uso de psicoestimulantes por estudiantes de medicina en un faculdad privado de Juiz de Fora. Se realizó un estudio observacional transversal con 213 estudiantes del 1 al $12^{\circ}$ semestre, excluidos los menores de 18 años. Los datos se recopilaron mediante el cuestionario: " Adult Self-Report Scale", donde se encontró el uso de psicoestimulantes en el 31,5\% de los entrevistados. Aún así, entre aquellos que no lo usaron, el $31.5 \%$ reportó interés previo en el uso. La mayoría de los usuarios eran mujeres (52.2\%), y $62.7 \%$ de los estudiantes que afirmaron usar psicoestimulantes no tenían un diagnóstico previo de trastorno por déficit de atención con hiperactividad (TDAH). Por lo tanto, se observa la relevancia de comprender el patrón de uso actual de los psicoestimulantes por parte de los estudiantes, corroborando las posibles estrategias para mejorar la suya salud mental.

Palabras clave: Trastorno por Déficit de Atención con Hiperactividad, Estudiantes, Epidemiología.

\section{INTRODUÇÃO}

Segundo a Associação Brasileira de Déficit de Atenção, o Transtorno de Déficit de Atenção e Hiperatividade (TDAH) é um distúrbio neurobiológico que tem como principal característica um padrão persistente de desatenção, inquietude e impulsividade. É de causa genética e geralmente aparece na infância, podendo acompanhar o indivíduo por toda a vida. Nos adultos, a hiperatividade pode se manifestar como inquietude extrema, dentre outros sintomas (AMERICAN PSYCHIATRIC ASSOCIATION, 2013).

É sabido que, classicamente, o TDAH acomete principalmente crianças, onde o diagnóstico é essencialmente clínico. Nos adultos observa-se dificuldades organizacionais; baixa eficiência em suas atividades cognitivas e/ou laborais; falhas consumadas em atividades acadêmicas ou de trabalho. Há de se notar também que o TDAH na vida adulta possui um forte componente genético, de forma que há congruência com anormalidades estruturais no cérebro. Outrossim, cabe ressaltar que a prevalência global estimada de TDAH na infância gira em torno de $5 \%$, enquanto na vida adulta cai para $2,5 \%$, com idade média de acometimento entre 19.4-44.9 anos (SIMON V, et al., 2009).

Pontua-se também que há uma associação negativa entre prevalência de TDAH na vida adulta e o envelhecimento, onde há uma progressão na redução dos sintomas (SIMON V, et al., 2009). Ainda, a proporção de diagnósticos na infância é de cerca de 1:1 em indivíduos do sexo masculino:feminino. Tal achado é atribuído ao fato de que na vida escolar a observação e o diagnóstico são feitos por pais ou professores, enquanto na vida adulta o diagnóstico é feito a partir da procura do paciente pelo médico - assim sendo, em adultos, a prevalência é maior em mulheres do que homens (3:1), pois elas procuram auxílio à saúde com maior frequência. Ainda, a prevalência de TDAH em adultos brasileiros é de 5,8\% (POLANCZYK GV, 2008).

No que diz respeito à terapia da desordem, tem-se como pedras angulares a intervenção médica, reestruturações ambientais, educacionais e comportamentais. Sobre a primeira, tem-se como opções terapêuticas mais disponíveis o metilfenidato e a lisdexanfetamina. Tais princípios ativos, marcados pela psicoestimulação gerada por anfetaminas, apresentam efeitos indesejáveis como distúrbios do sono e inapetência. Ainda, como terapias complementares, são recomendadas atividades ocupacionais, como práticas musicais e desportivas, além de exercícios de relaxamento. Há de se ressaltar também a fundamental importância da psicoterapia e suporte pedagógico que supram as demandas da hiperatividade e da perda de atenção (STUHEC M, et al., 2019).

O TDAH tem como primeira opção terapêutica medicamentosa o metilfenidato, um medicamento psicoestimulante que também pode ser usado no tratamento da narcolepsia e obesidade $A$ sua venda é controlada por meio de receituário especial, que visa limitar o acesso da população ao fármaco. Esse medicamento tem poucos efeitos colaterais, dos quais entre os de curto prazo prevalecem redução do apetite, insônia, cefaleia e dor abdominal, sendo a maioria autolimitada, dose dependente e de média intensidade. Já os de longo prazo são constatados por leves alterações de pressão arterial e na frequência cardíaca. (ORTEGA F, et al., 2010). 
Diante de tal contexto, é possível observar que acadêmicos de medicina são um dos principais alvos de estudos acerca de uso inadequado de psicoestimulantes. Elucidando isso, Urrego MA, et al. (2008), em pesquisa com 309 estudantes da Universidade de Manizales, demonstrou que 54 destes cursavam medicina, dentre os quais $42,3 \%$ afirmaram fazer uso de anfetaminas, classe na qual o metilfenidato se inclui. Por outro lado, 52 participantes do estudo eram alunos de direito, dos quais apenas 12,2\% reportaram uso. Para Carneiro SG, et al. (2013), isso provavelmente é justificado pelo grande esforço exigido pelo curso de medicina, que muitas vezes demanda várias horas de estudo e uma grande quantidade de conteúdo a ser memorizado. Além disso, os acadêmicos de medicina estão mais vulneráveis a transtornos mentais, sendo que, em um estudo de Moutinho ILD, et al. (2016), com acadêmicos da Universidade Federal de Juiz de Fora (UFJF), quase $50 \%$ destes apresentou níveis elevados de sintomas de depressão, ansiedade e estresse.

Dessa forma, o presente estudo teve como objetivo verificar o uso de psicoestimulantes por parte dos acadêmicos de medicina de uma instituição particular do município de Juiz de Fora - MG.

\section{MÉTODOS}

Foi realizado um estudo transversal para avaliar as prevalências do uso de psicoestimulantes e do TDAH entre um grupo de estudantes de medicina. Além disso, por meio de uma revisão bibliográfica, foram comparadas tais prevalências com medidas encontradas em outros estudos. Após a aprovação pelo Comitê de Ética em Pesquisa (CEP) da instituição, com funcionamento regular junto ao sistema da Comissão Nacional de Ética em Pesquisa (CONEP) e sob o parecer número 2.382.448, os dados foram coletados. Ademais fora obedecida a Resolução do Conselho Nacional de Saúde (CNS) 466/12.

Os participantes desta pesquisa assinaram um Termo de Consentimento Livre e Esclarecido (TCLE) previamente. Um psiquiatra esteve à disposição dos participantes em caso de necessidade, com manutenção de sigilo durante toda a pesquisa.

A coleta de dados foi feita entre fevereiro a maio de 2018. A população pesquisada foi estudantes de medicina do $1^{\circ}$ ao $12^{\circ}$ períodos, de uma faculdade de Juiz de Fora. Essa coleta foi feita através de dois questionários, sendo um geral e outro validado para auxílio diagnóstico de TDAH, conhecido como "Adult Self-Report Scale" (ASRS).

O questionário geral, elaborado pelos pesquisadores, é formado por 44 perguntas e dividido em cinco partes. É composto por fatores socioeconômicos, hábitos e vícios, comportamento e convívio social, uso de psicoestimulantes e diagnóstico prévio de TDAH. Já o ASRS tem valor preditivo fortemente positivo para TDAH. A partir das respostas obtidas pelo preenchimento dos questionários foi relacionado o uso do metilfenidato por estudantes de medicina ao diagnóstico prévio ou não de TDAH.

Os entrevistados possuem mais de 18 anos e são acadêmicos de medicina. Os alunos foram abordados aleatoriamente na instituição a fim de evitar o viés de seleção.

No que diz respeito ao espaço amostral, é desejável que um estudo do tipo transversal para estimar uma prevalência com precisão relativa, tendo em conta uma população de 600 alunos, um nível de significância de $95 \%$, uma frequência esperada do fator em estudo de $25 \%$ e um erro amostral de $5 \%$, o tamanho da amostra necessária é de aproximadamente 195 alunos.

Os dados foram armazenados no programa Access 2013, Microsoft Corporation® USA. Para a análise estatística foi utilizado o programa Statistical Package for the Social Sciences (SPSS) 21.0, licenciado e distribuído pela International Business Machines Corporation (IBM) IBM®SPSS Statistic. Medidas de posição e tendência central foram utilizadas para a descrição de variáveis contínuas e proporções para as variáveis categóricas estudadas.

$\mathrm{Na}$ análise bivariada foram verificadas diferenças entre variáveis contínuas através do teste T de igualdade de duas amostras independentes. Em variáveis categóricas para verificar diferenças entre duas amostras independentes foi utilizado o teste de qui-quadrado. 
Nos testes não paramétricos em variáveis quantitativas, após verificar a normalidade através do teste de Shapiro-Wilk, foram investigadas diferenças em amostras independentes com os testes de Mann-Whitney ou Kruskal-Wallis, dependendo do número das mesmas. Já em amostras relacionadas foram utilizados os testes de Wilcoxon ou Friedman em duas ou mais amostras, respectivamente. Em termos de análise do p-valor e dos intervalos de confiança o valor crítico foi definido em $95 \%$.

\section{RESULTADOS}

Foram avaliados um total de 213 alunos. Dos entrevistados, 59,2\% eram do sexo masculino $(n=126)$, possuíam idade média de 24,3 anos e $40,4 \%$ ( $n=86)$ moravam sozinhos. Destes, 33,8\% ( $n=72)$ estavam matriculados entre $\circ 9^{\circ}$ e $\circ 12^{\circ}$ períodos (Tabela 1).

Tabela 1 - Distribuição dos estudantes incluídos no estudo de acordo com dados socioeconômicos e período acadêmico.

\begin{tabular}{lcc}
\hline Variável & $\mathbf{N}$ & $\%$ \\
\hline Masculino & 126 & 59.2 \\
Branca & 162 & 76.1 \\
Católica & 178 & 83.6 \\
Solteiro & 206 & 96.7 \\
Mora sozinho & 86 & 40.4 \\
\hline Média de Idade & & \\
\hline$<20$ & 43 & 20.2 \\
$20-25$ & 94 & 44.1 \\
$>25$ & 76 & 35.7 \\
\hline Período & & \\
\hline $1^{\circ}-3^{\circ}$ & 68 & 31.9 \\
$4^{\circ}-5^{\circ}$ & 40 & 18.8 \\
$6^{\circ}-8^{\circ}$ & 33 & 15.5 \\
$9^{\circ}-12^{\circ}$ & 72 & 33.8 \\
\hline
\end{tabular}

Fonte: Teixeira AB, et al., 2020.

Em relação ao diagnóstico prévio de TDAH, 11,7\% $(n=25)$ o possuíam. Destes pacientes com déficit de atenção confirmado, $24 \%(n=6)$ também apresentavam outra doença psiquiátrica, como ansiedade e depressão (Tabela 2).

Tabela 2 - Relação entre doença psiquiátrica, diagnóstico prévio de TDAH e uso de psicoestimulantes por acadêmicos de medicina.

\begin{tabular}{|c|c|c|c|c|c|c|c|}
\hline \multirow{2}{*}{ Variáveis } & \multicolumn{4}{|c|}{ Diagnóstico de doença psiquiátrica } & & \multirow{2}{*}{ Total } & \multirow{2}{*}{$\begin{array}{c}\text { p- } \\
\text { valor }\end{array}$} \\
\hline & & im & & Não & & & \\
\hline \multicolumn{8}{|c|}{ Diagnóstico prévio de TDAH } \\
\hline Sim & 6 & $24.0 \%$ & 19 & $10.1 \%$ & 25 & $11.7 \%$ & 0,043 \\
\hline Não & 19 & $76.0 \%$ & 169 & $89.9 \%$ & 188 & $88.3 \%$ & \\
\hline \multicolumn{8}{|c|}{ Uso de psicoestimulante } \\
\hline Sim & 10 & $40.0 \%$ & 57 & $30.3 \%$ & 67 & $31.5 \%$ & 0,327 \\
\hline Não & 15 & $60.0 \%$ & 131 & $69.7 \%$ & 146 & $68.50 \%$ & \\
\hline
\end{tabular}

Fonte: Teixeira AB, et al., 2020.

Quando avaliados de acordo com a escala ASRS, $72,3 \%(n=154)$ dos alunos possuíam provável diagnóstico. A maioria dos acadêmicos com provável diagnóstico de TDAH é do sexo masculino (61\%), possui entre 20 e 25 anos $(40,3 \%)$ e cursa do $1^{\circ}$ ao $3^{\circ}$ períodos $(35,7 \%)$ (Tabela 3 ). 
Tabela 3 - Estatísticas descritíveis entre o provável diagnóstico de TDAH em relação ao sexo, idade, período acadêmico, tempo de concentração nos estudos, uso de psicoestimulantes e diagnóstico prévio de TDAH dos estudantes entrevistados.

\begin{tabular}{|c|c|c|c|c|c|c|c|}
\hline \multirow{2}{*}{ Variáveis } & \multicolumn{4}{|c|}{ Provável diagnóstico de TDAH } & \multirow{2}{*}{\multicolumn{2}{|c|}{ Total }} & \multirow{2}{*}{$\begin{array}{c}\mathbf{p}- \\
\text { valor }\end{array}$} \\
\hline & \multicolumn{2}{|c|}{ Sim } & \multicolumn{2}{|r|}{ Não } & & & \\
\hline \multicolumn{8}{|l|}{ Sexo } \\
\hline Feminino & 60 & $39.0 \%$ & 27 & $45.8 \%$ & 87 & $40.8 \%$ & \multirow{2}{*}{0,366} \\
\hline Masculino & 94 & $61.0 \%$ & 32 & $54.2 \%$ & 126 & $59.2 \%$ & \\
\hline \multicolumn{8}{|l|}{ Idade } \\
\hline$<20$ anos & 39 & $25.3 \%$ & 4 & $6.8 \%$ & 43 & $20.2 \%$ & \multirow{3}{*}{0,019} \\
\hline 20-25 anos & 62 & $40.3 \%$ & 32 & $54.2 \%$ & 94 & $44.1 \%$ & \\
\hline$>25$ anos & 53 & $34.4 \%$ & 23 & $69.0 \%$ & 76 & $35.7 \%$ & \\
\hline \multicolumn{8}{|l|}{ Períodos } \\
\hline $1^{\circ}-3^{\circ}$ & 55 & $35.7 \%$ & 13 & $22.0 \%$ & 68 & $31.9 \%$ & \multirow{4}{*}{0,265} \\
\hline $4^{\circ}-5^{\circ}$ & 26 & $16.9 \%$ & 14 & $23.7 \%$ & 40 & $18.8 \%$ & \\
\hline $6^{0}-8^{\circ}$ & 26 & $14.9 \%$ & 10 & $16.9 \%$ & 33 & $15.5 \%$ & \\
\hline $9^{\circ}-12^{\circ}$ & 50 & $32.5 \%$ & 22 & $37.3 \%$ & 72 & $33.8 \%$ & \\
\hline \multicolumn{8}{|c|}{ Diagnóstico prévio } \\
\hline Sim & 24 & $15.6 \%$ & 1 & $1.7 \%$ & 25 & $11.7 \%$ & \multirow{2}{*}{0,005} \\
\hline Não & 130 & $84.4 \%$ & 58 & $98.3 \%$ & 188 & $88.0 \%$ & \\
\hline \multicolumn{8}{|c|}{ Uso de psicoestimulantes } \\
\hline Sim & 52 & $33.8 \%$ & 15 & $25.4 \%$ & 67 & $31.5 \%$ & \multirow{2}{*}{0,240} \\
\hline Não & 102 & $66.2 \%$ & 44 & $74.6 \%$ & 146 & $68.5 \%$ & \\
\hline \multicolumn{8}{|c|}{ Tempo de concentração nos estudos } \\
\hline$\leq 45$ minutos & 73 & $47.4 \%$ & 21 & $35.6 \%$ & 94 & $44.1 \%$ & \multirow{2}{*}{0,120} \\
\hline$>45$ minutos & 81 & $52.6 \%$ & 38 & $64.4 \%$ & 119 & $55.9 \%$ & \\
\hline
\end{tabular}

Fonte: Teixeira AB, et al., 2020.

Quanto ao uso de psicoestimulantes, 31,5\% ( $n=67)$ dos entrevistados já fizeram uso, sendo que, dos que não utilizaram, $31,5 \%(n=47)$ já sentiram vontade. A maioria dos alunos que fez uso era do sexo feminino (52,2\%), possuía mais de 25 anos (49,3\%) e estava no internato, ou seja, do $9^{\circ}$ ao $12^{\circ}$ períodos $(37,3 \%)$.

Ainda em relação aos que usaram esses medicamentos, 62,7\% $(n=42)$ não possuíam diagnóstico prévio de TDAH. Porém, considerando a escala ASRS, 77,6\% $(n=52)$ possuíam um possível diagnóstico.

A melhora do rendimento acadêmico para provas foi manifestada em $53,7 \%$ ( $n=36)$ como o principal motivo para o uso, dado que $53,7 \%(n=36)$ relataram conseguir se concentrar nos estudos por, no máximo, 45 minutos. Apenas $37,3 \%(n=25)$ dos entrevistados utilizavam psicoestimulantes pelo diagnóstico de TDAH (Tabela 4). 
Tabela 4 - Frequência do uso de psicoestimulantes em relação ao sexo, idade, período acadêmico, tempo de concentração nos estudos, motivo de uso e diagnóstico prévio ou de possível de TDAH dos estudantes entrevistados.

\begin{tabular}{|c|c|c|c|c|c|c|c|}
\hline \multirow{2}{*}{ Variáveis } & \multicolumn{4}{|c|}{ Uso de psicoestimulante } & \multirow{2}{*}{\multicolumn{2}{|c|}{ Total }} & \multirow{2}{*}{ p-valor } \\
\hline & \multicolumn{2}{|c|}{ Sim } & \multicolumn{2}{|c|}{ Não } & & & \\
\hline \multicolumn{8}{|l|}{ Sexo } \\
\hline Feminino & 35 & $52.2 \%$ & 52 & $35.6 \%$ & 87 & $40.8 \%$ & \multirow{2}{*}{0,016} \\
\hline Masculino & 32 & $47.8 \%$ & 94 & $64.4 \%$ & 126 & $59.2 \%$ & \\
\hline \multicolumn{8}{|l|}{ Idade } \\
\hline$<20$ anos & 10 & $14.9 \%$ & 33 & $22.6 \%$ & 43 & $20.2 \%$ & \multirow{3}{*}{0,019} \\
\hline $20-25$ anos & 24 & $35.8 \%$ & 70 & $47.9 \%$ & 94 & $44.1 \%$ & \\
\hline$>25$ anos & 33 & $49.3 \%$ & 43 & $29.5 \%$ & 76 & $35.7 \%$ & \\
\hline \multicolumn{8}{|l|}{ Períodos } \\
\hline $1^{\circ}-3^{\circ}$ & 18 & $26.9 \%$ & 50 & $34.2 \%$ & 68 & $31.9 \%$ & \multirow{4}{*}{0,171} \\
\hline $4^{\circ}-5^{\circ}$ & 17 & $25.4 \%$ & 23 & $15.8 \%$ & 40 & $18.8 \%$ & \\
\hline $6^{\circ}-8^{\circ}$ & 7 & $10.4 \%$ & 26 & $17.8 \%$ & 33 & $15.5 \%$ & \\
\hline $9^{\circ}-12^{\circ}$ & 25 & $37.3 \%$ & 47 & $32.2 \%$ & 72 & $33.8 \%$ & \\
\hline \multicolumn{8}{|c|}{ Diagnóstico prévio } \\
\hline Sim & 25 & $37.3 \%$ & - & - & 25 & $11.7 \%$ & \\
\hline Não & 42 & $62.7 \%$ & 146 & $100 \%$ & 188 & $88.3 \%$ & \\
\hline \multicolumn{8}{|c|}{ Possível diagnóstico } \\
\hline Sim & 52 & $77.6 \%$ & 102 & $69.9 \%$ & 154 & $72.3 \%$ & \multirow{2}{*}{0,241} \\
\hline Não & 15 & $22.4 \%$ & 44 & $30.1 \%$ & 59 & $27.7 \%$ & \\
\hline \multicolumn{8}{|c|}{ Tempo de concentração nos estudos } \\
\hline$\leq 45$ minutos & 36 & $53.7 \%$ & 58 & $39.7 \%$ & 94 & $44.1 \%$ & \multirow{2}{*}{0,056} \\
\hline$>45$ minutos & 31 & $46.3 \%$ & 88 & $60.3 \%$ & 119 & $55.9 \%$ & \\
\hline \multicolumn{8}{|c|}{ Motivo do uso } \\
\hline TDAH & 25 & $37.3 \%$ & - & - & - & - & \multirow{4}{*}{ - } \\
\hline Vestibular & 5 & $7.5 \%$ & - & - & - & - & \\
\hline Provas & 36 & $53.7 \%$ & - & - & - & - & \\
\hline Outro & 1 & $1.5 \%$ & - & - & - & - & \\
\hline
\end{tabular}

Fonte: Teixeira AB, et al., 2020.

\section{DISCUSSÃO}

Este estudo proporciona informações sobre a prevalência do uso de psicoestimulantes entre os universitários do curso de medicina de uma faculdade particular de Juiz de Fora, tendo relevância ao contribuir com dados sobre a utilização dessas drogas dissociada do diagnóstico de TDAH. Segundo Itaborahy C (2009); até o ano de 2009 não havia estudos publicados sobre o uso não prescrito do metilfenidato no Brasil.

É lamentável ressaltar que a prática de doping mental, na forma de neuroaprimoramento farmacológico torna-se, cada vez mais, uma prática frequente entre os universitários, sendo que tal prática é observada em cerca de 5 a 7\% dos universitários dos Estados Unidos da América. É digno de nota que os motivos para o uso abusivo de drogas apontam para outros comportamentos em relação ao uso de outras substâncias. A exemplo disto, cita-se o álcool sendo usado nocivamente como modulador de humor (SMITH ME e FARAH MJ, 2011).

No presente estudo, observou-se que $31,5 \%$ dos acadêmicos já utilizaram algum tipo de psicoestimulante, sendo que, destes, 62,7\% não possuíam diagnóstico de TDAH. Em comparação, em uma população de acadêmicos de medicina de uma Faculdade do Sul de Minas Gerais, foi encontrada prevalência de 29,16\% do uso de metilfenidato. Destes, apenas $4,16 \%$ relataram uso prescrito para tratamento de TDAH, sendo $25 \%$ a prevalência de uso do metilfenidato sem prescrição médica. Quando avaliados estudantes em geral, no Brasil, esse valor foi de $28,4 \%$ em um universo de 36.588 (LAGE DC, et al., 2015).

Portanto, cabe pontuar o fato de que, no presente estudo, fora observada uma prevalência maior de estudantes que já fizeram uso de neuroaprimoradores farmacológicos quando comparados à outros dados 
da Literatura, em que 9,8\% dos estudantes das três grandes áreas de uma Universidade fizeram uso de alguma droga off label para tal finalidade (CÂNDIDO RC, et al., 2020).

Tal discrepância pode ser atribuída ao fato de que os acadêmicos de medicina estão expostos à uma carga horária e pressão social além do usual em outros cursos de graduação. Ademais, há de se ressaltar também a facilidade do acesso às medicações por parte dos estudantes do curso, tanto pelo poder aquisitivo quanto pelo contato cotidiano com o sistema de saúde em todas suas dimensões (FIOROTTI KP, et al., 2010).

Na pesquisa realizada, $53,7 \%$ dos acadêmicos que utilizaram metilfenidato alegaram que a melhora do rendimento para provas era o principal motivo para o uso. Na Universidade Federal da Bahia, em um estudo realizado com 186 alunos, $87,5 \%$ indicaram aumento da produtividade escolar como razão para o uso da substância (CRUZ TCSC, et al., 2011).

Silveira RR, et al. (2014), detectou uma maior prevalência do uso do metilfenidato entre estudantes em períodos mais avançados da graduação, corroborando achados do presente estudo, que constatou que a maioria $(37,3 \%)$ dos acadêmicos envolvidos no estudo utilizavam medicações como metilfenidato e lisdexanfetamina estão cursando os últimos anos da faculdade de medicina.

Apesar de não ser uma prática bem aceita no universo acadêmico, Greely H, et al. (2008), publicou um artigo contra argumentando as críticas. O mesmo defende o uso não prescrito de fármacos para aprimoramento cognitivo como uma nova prática para melhorar o desempenho, colocando-os na mesma posição de atividades rotineiras como uso de cafeína e prática de alimentação saudável. Os achados desse estudo corroboram com o que já está consolidado na Literatura, de forma que tem se tornado prática entre os universitários o uso de metilfenidato como doping intelectual (TETER CJ, et al, 2006).

De tal forma, há de se voltar a atenção para essa parcela da população, com medidas que visem a prevenção do uso e abuso de tais drogas, alertando para a importância da manutenção de bons hábitos e satisfatória qualidade de vida. É almejado que seja explicitado aos usuários sobre os efeitos adversos e malefícios de tais práticas, como por exemplo, o agravamento de sinais e sintomas de psicose ou mania (CÂNDIDO RC, et al., 2020). Estima-se que 0,10\% dos pacientes em uso de metilfenidato apresenta episódios de surtos psicóticos (MORAN LV, et al., 2019).

Outro ponto que merece atenção é o uso recreativo e abusivo de psicoestimulantes. Trata-se de uma situação de alerta, pois, é sabido que a ingestão de álcool associada à anfetaminas leva o indivíduo à uma maior ingesta de etanol, bem como um aumento da estimulação locomotora e ataxia, sensação de euforia e energia, além de uma falsa impressão de que o usuário não se encontra ébrio - há relatos que apontam para a mesma sensação de uso de álcool concomitante à cocaína (BARKLA XM, et al,. 2015).

Diante disso, torna-se imprescindível uma vigilância e situação de alerta para tal condição, em que o indivíduo toma uma postura nociva para si, o que vai além dos efeitos adversos de anfetaminas, tais como síndrome serotoninérgica ou distúrbios pressóricos e cardiovasculares, e também para a sociedade que o cerca, dadas as consequências catastróficas do uso abusivo de etanol, tanto pelo binge drinking quanto pelo etilismo por si só (TETER CJ, et al., 2006). É essencial que sejam adotadas estratégias educacionais e políticas de prevenção para o psicoaprimoramento com psicofármacos, com enfoque no fato de que, tal prática, aparentemente inócua, possui riscos e consequências, devendo ser evitada.

Exposto tal contexto, é válido angariar razões e causas para tal efeito entre os jovens adultos e acadêmicos. Os principais motivos para essa prática preocupante entre os universitários são as alegações de desejo por melhoria na concentração e no rendimento dos estudos, bem aumento do tempo em vigília e, por vezes, até mesmo para experimentar a sensação trazida pela droga. Ademais, há de se destacar que a maioria dos estudantes inicia seu uso de anfetaminas após o advento ao ambiente acadêmico (TETER CJ, et al., 2006).

Portanto, é cabível corroborar com trabalhos que visem a melhoria na qualidade de vida e dos estudos de acadêmicos de medicina, os quais usualmente apresentam altos índices de transtornos de humor, com prevalência entre 21,9\% - 30,6\% (VASCONCELOS TC, et al., 2015). 
É compreensível que os fatores que compõem sua esfera biopsicossocial vão sendo minados durante o processo de sua graduação, enquanto os sentimentos de aflição e pressão social culminam, dentre outros desfechos, na busca constante por melhorias, sendo a aprimoração farmacológica uma delas. Outrossim, há de se refletir sobre o espaço acadêmico e o comportamento almejado com o psicoaprimoramento, em que ambas possuem entre si o cerne da competitividade, cuja resultante comportamental usualmente é a busca e uso inadvertido e inapropriado de anfetaminas. Isso é corroborado pelo fato de que a maioria dos estudantes que apresenta tal prática, deu início ao uso das medicações após sua entrada na faculdade (TETER TC, et al., 2006). Destaca-se também o papel do efeito placebo, em que a simples expectativa pelo uso da droga traz consigo mudanças no humor e na perspectiva do estudante em relação às suas obrigações acadêmicas (LOOBY A e EARLEYWINE M, 2011).

Ademais, é desejável que mais trabalhos sejam conduzidos para a exploração do tema, como por exemplo a avaliação da performance acadêmica, ou ponderar acerca das formas de aquisição das drogas de abuso, explicitando se foram por vias lícitas ou não. Há ainda de se ressaltar sobre o comportamento usualmente desafiador do jovem, em que a busca por um objeto novo e supostamente inalcançável corroboram para atitudes impulsivas, como o uso abusivo e inadvertido de substâncias, lícitas ou não (BORBA MP e UBRAIS A, 2017).

Este estudo apresenta algumas limitações na avaliação do uso de psicoestimulantes, tendo em vista que este foi referido pelos participantes, dependendo diretamente de sua sinceridade nas respostas. A principal restrição do nosso estudo, por ter desenho transversal, é o fato de que os dados podem não ser representativos de todos os estudantes de medicina do Brasil. Todavia, já está claro que o uso não prescrito de psicoestimulantes é mais do que uma realidade no Brasil.

\section{CONCLUSÃO}

No presente estudo concluiu-se que psicoestimulantes foram usados por $31,5 \%$ ( $n=67)$ do total de acadêmicos investigados e $62,7 \%(n=42)$ dos que reportaram uso não possuíam diagnóstico de TDAH. A continuidade dos estudos sobre esse tema se faz necessária para o aumento do volume de dados e a solidificação das evidências. O uso de psicoestimulantes de maneira inadequada e/ou sem diagnóstico prévio pode acarretar em diversos impactos na vida de quem o faz. Os dados deste estudo podem auxiliar educadores no planejamento de estratégias que melhorem a saúde mental e a prevenção do uso de psicoestimulantes de forma incorreta pelos estudantes de medicina.

\section{REFERÊNCIAS}

1. AMERICAN PSYCHIATRIC ASSOCIATION. Diagnostic and statistical manual of mental disorders (DSM-5®). American Psychiatric Pub; 2013.

2. BARKLA XM, et al. Are there any potentially dangerous pharmacological effects of combining ADHD medication with alcohol and drugs of abuse? A systematic review of the literature. BMC psychiatry. 2015; 15(1): 270.

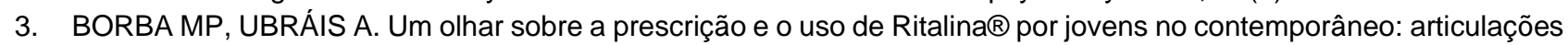
sobre corpo, liberdade, poder e subjetividade. Corpos, poderes e processos de subjetivação: discursos e práticas na cultura contemporânea. 2017: 229.

4. CÂNDIDO RC, et al. Prevalência e fatores associados ao uso de metilfenidato para neuroaprimoramento farmacológico entre estudantes universitários. Einstein (São Paulo). 2020;18.

5. CARNEIRO SG, et al. O uso não prescrito de metilfenidato entre acadêmicos de Medicina. Cadernos Uni-FOA: Edição Especial Ciênc da Saúde e Biol, 2013; 53-9.

6. CRUZ TCSC, et al. Uso não prescrito de metilfenidato entre estudantes de medicina da Universidade Federal da Bahia. Gazeta Médica da Bahia, 2011; 81(1): 3-6.

7. FIOROTTI KP, et al. Transtornos mentais comuns entre os estudantes do curso de medicina: prevalência e fatores associados. J Bras Psiquiatr. 2010;59(1):17-23.

8. GREELY H, et al. Towards responsible use of cognitive-enhancing drugs by the healthy. Nature 456.7223 (2008): 702-705. 
9. ITABORAHY C. A Ritalina no Brasil: uma década de produção, divulgação e consumo, RJ. Dissertação (Mestrado em Medicina) - Instituto de Medicina Social. Universidade do Estado do Rio de Janeiro, Rio de Janeiro, $2009 ; 126$ p.

10. LAGE DC, et al. Uso de metilfenidato pela população acadêmica: revisão de literatura. Brazilian Journal of Surgery and Clinical Research, 2015; 10(3): 31-9.

11. LOOBY A, EARLEYWINE M. Expectation to receive methylphenidate enhances subjective arousal but not cognitive performance. Experimental and clinical psychopharmacology. 2011 Dec;19(6):433.

12. MORAN LV, et al. Psychosis with Methylphenidate or Amphetamine in Patients with ADHD. New England Journal of Medicine. 2019 Mar 21;380(12):1128-38.

13. MOUTINHO ILD, et al. Depression, stress and anxiety in medical students: A cross-sectional comparision between students from diferent semesters. Rev. Assoc. Med. Bras, 2017; 63(01): 21-8.

14. ORTEGA F, et al. A ritalina no Brasil: produções, discursos e práticas. Intaface-Comunic. Saúde, Educação, 2010; 14(34): 499-510.

15. POLANCYK GV. Estudo da prevalência do Transtorno de Déficit de Atenção/Hiperatividade na infância, adolescência e idade adulta. Porto Alegre. Tese [Doutorado em Psiquiatria] - Universidade Federal do Rio Grande do Sul, Porto Alegre, 2008.

16. SILVEIRA RR, et al. Patterns of non-medical use of methylphenidate among 5th and 6th year students in a medical school in southern Brazil. Trends in Psychiatry and Psychotherapy, 2014; 36(02): 101-6.

17. SIMON V, et al. Prevalence and correlates of adult attention-deficit hyperactivity disorder: meta-analysis. The British Journal of Psychiatry. 2009 Mar;194(3):204-11.

18. SMITH ME, FARAH MJ. Are prescription stimulants "smart pills"? The epidemiology and cognitive neuroscience of prescription stimulant use by normal healthy individuals. Psychological bulletin. 2011 Sep;137(5):717.

19. STUHEC $M$, et al. Efficacy, acceptability, and tolerability of lisdexamfetamine, mixed amphetamine salts, methylphenidate, and modafinil in the treatment of attention-deficit hyperactivity disorder in adults: a systematic review and meta-analysis. Annals of Pharmacotherapy. 2019 Feb;53(2):121-33.

20. TETER CJ, et al. Illicit use of specific prescription stimulants among college students: prevalence, motives, and routes of administration. Pharmacotherapy. 2006;26(10):1501-10

21. URREGO MA, et al. Consumo de anfetaminas, para mejorar el rendimento académico, em estudiantes de la Universidad de Manizales. Arch Med, 2008; 9 (1): 43-57.

22. VASCONCELOS TC, et al. Prevalência de sintomas de ansiedade e depressão em estudantes de medicina. Revista Brasileira de Educação Médica. 2015 Mar;39(1):135-42. 